\title{
AMBIENTE VIRTUAL DE APRENDIZAGEM: ELEMENTOS E FERRAMENTAS QUE INFLUENCIAM A INTERAÇÃO ONLINE
}

\author{
VIRTUAL LEARNING ENVIRONMENT: \\ ELEMENTS AND TOOLS THAT INFLUENCIA ONLINE \\ INTERACTION
}

\section{ENTORNO VIRTUAL DE APRENDIZAJE: ELEMENTOS Y HERRAMIENTAS QUE INFLUENCIAN LA INTERACCIÓN ONLINE}

Josefa Kelly Cavalcante de Oliveira ${ }^{1}$

\begin{abstract}
RESUMO: Este estudo tem como objetivo analisar as interações e interatividade no ambiente virtual de aprendizagem (AVA) Moodle de uma disciplina do curso de Pedagogia - UAB da Universidade Federal de Alagoas, bem como identificar a concepção que alunado possui sobre a influência da interação no processo de ensino aprendizagem. Partindo do conceito que aprender envolve interação e participação de todos e que na $\mathrm{EAD}$, a mediação acontece por meio do AVA. A questão norteadora é a seguinte: O que motiva o aluno a interagir no AVA e quais as ferramentas e elementos disponíveis no AVA que influenciam a interação virtual? O objetivo é analisar o papel das ferramentas de interação como recurso para gerar motivação e fomentar aprendizagem colaborativa. O estudo teórico tem como base Behar (2013), Illera (2010), Mattar (2011), Silva (2017) entre outros autores que serviram de fundamentação teórica/bibliográfica para este estudo. A pesquisa de cunho qualitativo conforme Sampiere; Collado e Lúcio (2013), envolve estudo de caso segundo Yin (2015). O percurso metodológico envolveu coleta de dados por meio de questionários aplicados a alunos do curso de Pedagogia EAD. Os resultados permitiram perceber que os alunos compreendem a interação como elemento importante para a construção colaborativa do conhecimento e que as principais ferramentas de interação é o fórum e os espaços de postagem de atividades que servem como meio para troca de conhecimentos.
\end{abstract}

Palavras chave: ambientes virtuais, EAD, interação, interatividade, ferramentas digitais.

Submetido em: 22/11/2017 - Aceito em: 07/05/2018 - Publicado em: 30/06/2018.

${ }^{1}$ Graduada em Pedagogia (Universidade Federal de Alagoas - UFAL) e Mestranda em Educação pelo Programa de Pós-Graduação em Educação de Alagoas (Universidade Federal de Alagoas - UFAL). Email: pedagoga.kellycavalcante@gmail.com 


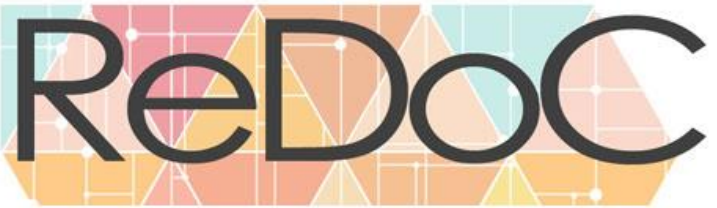

Revista Docência e Cibercultura

ABSTRACT: This study aims to analyze interactions and interactivity in the virtual learning environment (AVA) Moodle of a course of Pedagogy - UAB of the Federal University of Alagoas, as well as to identify the conception it has on the influence of interaction in the process of teaching learning. Starting from the concept that learning involves interaction and participation of all and that in the EAD, mediation takes place through the AVA. The guiding question is: What motivates the student to interact in the AVA and what tools and elements are available in the AVA that influence the virtual interaction? The objective is to analyze the role of interaction tools as a resource to generate motivation and foster collaborative learning. The theoretical study is based on Behar (2013), Illera (2010), Mattar (2011), Silva (2017) among other authors that served as theoretical / bibliographic basis for this study. The qualitative research according to Sampiere; Collado and Lucio (2013), involves a case study according to Yin (2015). The methodological course involved data collection through questionnaires applied to students of the EAD Pedagogy course. The results allowed us to understand that students understand interaction as an important element for the collaborative construction of knowledge and that the main interaction tools are the forum and the posting spaces of activities that serve as a medium for knowledge exchange.

Key words: virtual environments, EAD, interaction, interactivity, digital tools.

RESUMÉN: Este estudio tiene como objetivo analizar las interacciones e interactividad en el entorno virtual de aprendizaje EVA Moodle de una disciplina del curso de Pedagogía -UAB de la Universidad Federal de Alagoas, como también identificar la concepción que el alumnado posee sobre la influencia de la interacción en el proceso de enseñanza aprendizaje. Partiendo del concepto que aprender comprende interacción y participación de todos y que en la EaD la mediación se da por medio del EVA. La cuestión disparadora es la siguiente: ¿Qué motiva al alumno a interactuar en el AVA y cuáles son las herramientas y elementos disponibles en el EVA que influencian la interacción virtual? El estudio teórico tuvo como base Behar (2013), Illera (2010), Mattar (2011), Silva (2017) entre otros autores que sirvieron de fundamentación teórica/bibliográfica para este estudio. El desarrollo metodológico realizó la colecta de datos por medio de cuestionarios aplicados a alumnos del curso de Pedagogía EaD. Los resultados permitieron percibir que los alumnos comprenden la interacción como elemento importante para la construcción colaborativa del conocimiento y que las principales herramientas de interacción son el correo electrónico, foro y los espacios de publicación de actividades que sirven como medio de intercambio de conocimientos.

Palabras clave: entornos virtuales, EAD, interacción, interactividad, herramientas digitales. 


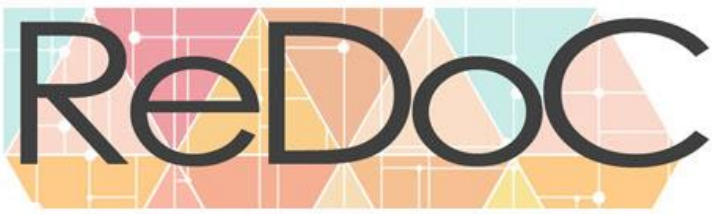

Revista Docência e Cibercultura

\section{INTRODUÇÃO}

Segundo Santos (2002) a Educação a Distância é uma modalidade de ensino na qual professores e alunos estão geograficamente separados e em tempos distintos. Nesse contexto, os ambientes virtuais de aprendizagem vêm se estabelecendo a partir do uso criativo das TDIC. Estes espaços podem proporcionar ao alunado um ambiente rico em estímulos cognitivos e aprendizagem colaborativa. Para isso, é preciso que a interface, os materiais educacionais didáticos e a motivação para aprender proporcionem interação entre os envolvidos.

Os AVAs, vão além da representação da sala de aula presencial, pois englobam o uso de mídias e recursos que permitem que o ensino-aprendizagem ultrapasse barreiras geográficas e de tempo. Isto é, o ato de ensinar e aprender não se restringem a sala de aula física, com paredes de concretos, quadro negro e giz, pois ao se romper a distância e o tempo com o uso das Tecnologias Digitais da Informação e Comunicação-TDIC, ocorre uma mudança no modo de agir do professor e do aluno. Quer dizer, o professor torna-se um mediador de aprendizagem e o aluno o construtor do seu conhecimento.

Conforme Kenski (2004) o aluno por sua vez, ao torna-se mais responsável pela construção do seu aprendizado, tende a participar mais e a sentir-se integrado no processo de ensino-aprendizagem, além de expressar maior iniciativa, dividir o que sabe entre os demais, apresentar maior interesse, esforço de aprendizado e concentra-se melhor nas atividades realizadas. Todavia, para que isso aconteça é necessário que o professor conheça o contexto da aprendizagem virtual e que saiba utilizar/escolher objetos de aprendizagem e os materiais educacionais digitais, que tenham relação com o conteúdo a ser estudado. Afastando-se de métodos empregados no ensino presencial que não condizem com a aprendizagem em rede.

Subsequente, este estudo tem como objetivo analisar as interações e interatividade no AVA de uma disciplina do curso de Pedagogia UAB da Universidade Federal de Alagoas, bem como identificar a concepção que alunado possui sobre a influência da interação no processo de ensino aprendizagem. Partindo do conceito que aprender 


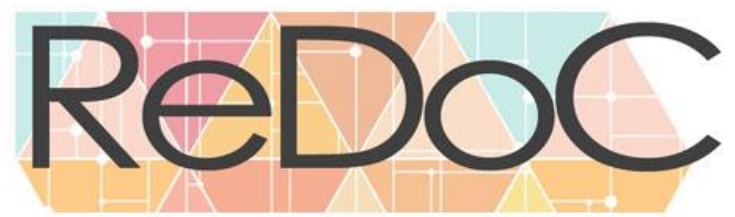

Revista Docência e Cibercultura

envolve interação e participação de todos, e que na EAD a mediação acontece por meio do AVA. A questão norteadora é a seguinte: o que motiva o aluno a interagir no AVA e quais as ferramentas e elementos disponíveis no AVA que influenciam a interação online? O estudo teórico tem como base Behar (2013), Illera (2010), Mattar (2011), Silva (2017) entre outros autores que serviram de fundamentação teórica/bibliográfica para este estudo. A pesquisa de cunho qualitativo conforme Sampiere; Collado e Lúcio (2013), envolve estudo de caso segundo Yin (2015). O percurso metodológico envolveu coleta de dados por meio de questionários aplicados a alunos do curso de Pedagogia EAD.

\section{INTERAÇÃO E INTERATIVIDADE EM AMBIENTES VIRTUAIS DE APRENDIZAGEM.}

O AVA proporciona o redimensionamento do ensinar e do aprender, o qual era realizado principalmente no espaço escolar. Marquesi e Araújo Jr (2008) apontam que o AVA deve potencializar a comunicação, permitindo interação e interatividade por meio dos recursos tecnológicos, além disso que as atividades a serem realizadas no AVA, deve constituir um percurso que promova a autonomia e a exploração dos espaços e recursos virtuais, permitindo uma aprendizagem significativa.

Contudo, para evitar a transposição de conteúdo e não tornar o AVA um espaço destinado ao armazenamento de atividades, é necessário compreender as mudanças que ocorreram na forma de adquirir conhecimento conforme a passagem do tempo, como especifica o quadro 1 a seguir:

Quadro 1: Mudanças na forma de adquirir conhecimento no decorrer do tempo.

\begin{tabular}{|l|l|}
\hline \multicolumn{2}{|c|}{ FORMAS DE ADQUIRIR CONHECIMENTO } \\
\hline \multicolumn{1}{|c|}{ SÉC XIX } & \multicolumn{1}{c|}{ SÉC XXI } \\
\hline O professor é o centro. & O centro do aprendizado é o aluno. \\
\hline $\begin{array}{l}\text { Valoriza o conteúdo. Exposição do } \\
\text { conteúdo. }\end{array}$ & $\begin{array}{l}\text { Valoriza a aprendizagem autônoma e } \\
\text { colaborativa. }\end{array}$ \\
\hline $\begin{array}{l}\text { Passividade do aluno. Assimilar } \\
\text { conteúdo. }\end{array}$ & $\begin{array}{l}\text { Participação ativa do aluno. Construir } \\
\text { conhecimento. }\end{array}$ \\
\hline
\end{tabular}




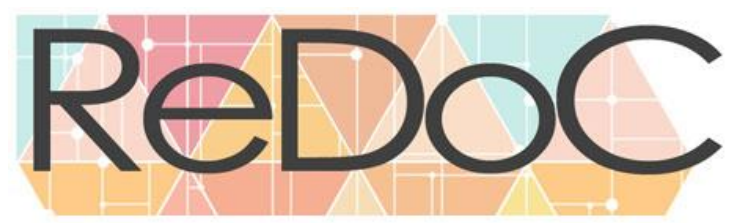

Revista Docência e Cibercultura

\begin{tabular}{|l|l|}
\hline Grande número de atividades. & $\begin{array}{l}\text { Atividades baseada em resoluções de } \\
\text { problemas e reflexivas. }\end{array}$ \\
\hline Individualidade & Colaboração \\
\hline Sequência linear & Hipertexto \\
\hline
\end{tabular}

Elaborado com base em: Illera (2010).

Com base no no quadro 1, percebe-se as mudanças que as TDIC proporcionaram ao campo educacional, posto que diante da sociedade globalizada na qual vivemos o individuo necessita de novas habilidades e competências para engajar-se frente aos desafios da sociedade. Nesse cenário as ações do docente e a postura do aluno mudam, àquele passa a ser um mediador de aprendizagem e este um indivíduo que constrói seu conhecimento.

Diante disso, Pereira, Schmitt e Dias (2007, p. 2) explicitam que: "O processo de ensino-aprendizagem tem potencial para tornar-se mais ativo, dinâmico e personalizado por meio de Ambientes Virtuais de Aprendizagem." Principalmente por este permitir uma abordagem mais atual, no qual o AVA por meio das TDIC promove a interação entre os participantes e interatividade com o conteúdo a ser aprendido. Contemplando também diferentes estilos de aprendizagem.

O AVA conta com essas ferramentas para promover a interação e melhorar a qualidade da aprendizagem na EAD. Por meio delas é possível esclarecer dúvidas, refletir e dialogar sobre os conteúdos, engajar-se com o grupo e contribuir com ideias, opiniões e conhecimento. A comunicação e interação acontece de várias formas dentro do AVA, dentre elas:

Quadro 2: Formas de interação no AVA.

\begin{tabular}{|l|l|}
\hline Mesmo tempo e lugares diferentes & $\begin{array}{l}\text { Compreende as ocasiões em que } \\
\text { professor e aluno estão separados } \\
\text { geograficamente, mas interagem de modo } \\
\text { síncrono. }\end{array}$ \\
\hline Tempos diferentes e no mesmo lugar & $\begin{array}{l}\text { Compreende as ocasiões assíncronas. } \\
\text { Utilização da mesma ferramenta de } \\
\text { comunicação, mas em tempo distinto. }\end{array}$ \\
\hline Tempos e lugares diferentes & $\begin{array}{l}\text { Compreende o acesso e o uso do AVA e a } \\
\text { relação com o material educacional }\end{array}$ \\
\hline
\end{tabular}




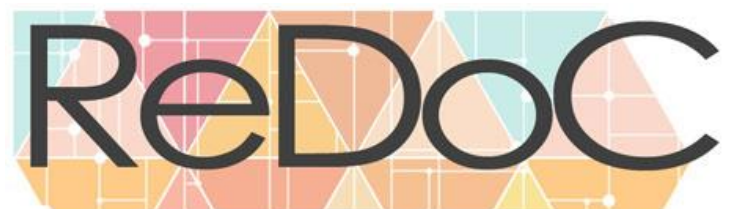

Revista Docência e Cibercultura

\begin{tabular}{|l|l|}
\hline & $\begin{array}{l}\text { didático e os demais recursos para a } \\
\text { aprendizagem. Utiliza a comunicação } \\
\text { assíncrona e síncrona. }\end{array}$ \\
\hline
\end{tabular}

Elaborado conforme: Belloni (2001).

$\mathrm{Na} \mathrm{EAD}$ as ferramentas tecnológicas podem ser assíncronas e/ou síncronas. São classificadas como síncronas as ferramentas de comunicação na qual a interação ocorre em horário especifico, com os envolvidos reunidos virtualmente no mesmo espaço de tempo, tais como: chat e videoconferência. A sincronicidade no AVA permite que os alunos se sintam mais próximos, atribui a sensação de grupo. As vantagens dessas ferramentas síncronas são:

- MOTIVAÇÃO: estimula a estudar e perseverar no curso.

- TELEPRESENÇA: comunicação e interação em tempo real. E troca de conhecimento concomitante.

- FEEDBACK: retorno das ações e dúvidas, bem como críticas construtivas instantaneamente.

- ENCONTRO COM O GRUPO: permite que o alunado se encontre, além de permitir o controle do tempo destinado as atividades. Lins, Moita e Dacol (2006).

Já as ferramentas assíncronas não necessitam de simultaneidade para que a comunicação aconteça, independem de tempo especifico, como o fórum e e-mail. A comunicação e a troca de mensagens ocorrem em tempos distintos. As vantagens dessas ferramentas assíncronas são:

- FLEXIBILIDADE: acessar a informação no tempo que melhor convém, dentro do prazo estipulado.

- REFLEXÃO: o aluno pode refletir sobre o conteúdo que está sendo discutido antes de dar sua contribuição. 


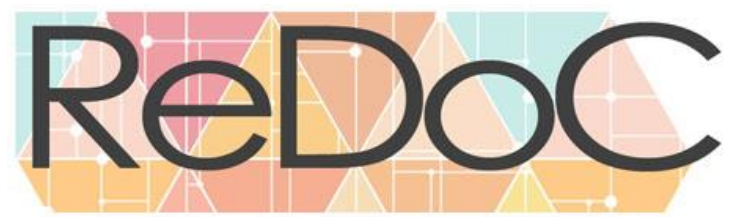

Revista Docência e Cibercultura

- CONEXÃO: problemas relacionados a conexão e velocidade do processador não irá prejudicar a participação e a contribuição na comunicação, pois será possível acessar em outro momento a discussão estabelecida.

- CONTEXTUALIZAÇÃO: buscar e analisar referências, além do recomendado, pensar sobre as respostas dos demais e sobre a sua. Lins, Moita e Dacol (2006).

As ferramentas apresentam características e formas distintas de uso que podem complementar e favorecer a aprendizagem por meio da colaboração e motivação. Conforme Silva e Figueiredo (2012) a interação envolve o contato humano. Relacionada a comunicação no AVA permite que um número de sujeitos se torne um grupo, no qual a ação individual no ambiente virtual torna-se um estimulo para o outro e vice-versa surgindo uma troca de experiencias e de conhecimento. Os autores acrescentam que a aprendizagem não se constitui em fragmentos e dissociada de relações pessoais, quer dizer, o conhecimento está relacionado a uma construção colaborativa. No ambiente virtual há diversos tipos de interações entre os sujeitos envolvidos como demostra o quadro 3 a seguir:

Quadro 3: Tipos de interações em Ambientes Virtuais de Aprendizagem.

\begin{tabular}{|l|l|}
\hline \multicolumn{1}{|c|}{ TIPOS DE } & \multicolumn{1}{c|}{ CARACTERÍSTICAS } \\
\hline Aluno-interface & $\begin{array}{l}\text { Exclusiva da EAD, envolve também interatividade. Refere- } \\
\text { se a forma como o aluno utiliza a tecnologia usada na } \\
\text { apresentação do conteúdo para aprender, mas também para } \\
\text { interagir com o professor e os demais. }\end{array}$ \\
\hline Aluno-professor & $\begin{array}{l}\text { Uma das interações mais importantes para obter resultados } \\
\text { significativos. Traz a importância do Feedback em tempo } \\
\text { hábil, para que o objetivo da mensagem seja compreendido } \\
\text { pelo aluno, antes que o mesmo perca o interesse pelo } \\
\text { conteúdo. Refere-se também a interação aluno-tutor. }\end{array}$ \\
\hline Aluno-aluno & $\begin{array}{l}\text { A interação aluno-aluno acontece de forma síncrona e/ou } \\
\text { assíncrona. Quando o ambiente virtual favorece essa } \\
\text { interação gera no grupo um sentimento de pertencimento e } \\
\text { permite a aprendizagem colaborativa e cooperativa. }\end{array}$ \\
\hline Auto interação & $\begin{array}{l}\text { Envolve os diálogos internos do aluno consigo mesmo. Suas } \\
\text { reflexões sobre o conteúdo e seu processo de construção da } \\
\text { aprendizagem. }\end{array}$ \\
\hline
\end{tabular}




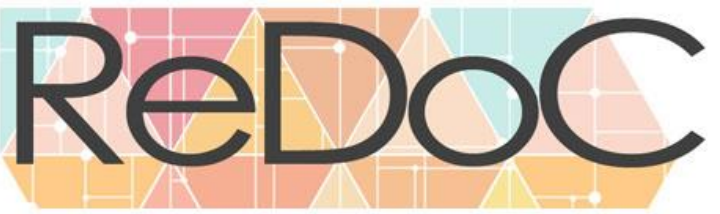

Revista Docência e Cibercultura

Adaptado de João Mattar (2011)

A expressão interatividade, por sua vez, está relacionada a interações não humanas Mattar (2011), mas que permite aos indivíduos não agir com passividade perante as ferramentas midiáticas. Em outras palavras, é a interação sujeito-objeto. Por conseguinte, a interatividade potencializa a presença virtual permitindo ao aluno cocriar $\mathrm{o}$ conhecimento em colaboração com os demais por meio das TDIC, Silva (2010). Trazendo o conceito de aproximação entre os indivíduos no ambiente virtual de aprendizagem. $\mathrm{O}$ quadro 3, aponta os tipos de interatividade sujeito-objeto.

Quadro 3: Tipos de interatividades em Ambientes Virtuais de Aprendizagem.

\begin{tabular}{|l|l|}
\hline \multicolumn{1}{|c|}{ TIPOS DE } & \multicolumn{1}{c|}{ CARACTERISTICAS } \\
\hline Aluno-interface & Interação entre o aluno e a tecnologia. \\
\hline Aluno-ferramenta & $\begin{array}{l}\text { Interação entre as ferramentas dentro e fora do AVA. } \\
\text { Ferramentas estas que enriquece a experiencia da EAD, } \\
\text { como câmeras, softwares, gravações e etc. }\end{array}$ \\
\hline Aluno-ambiente & Quando o aluno visita locais fora do ambiente virtual. \\
\hline Aluno-conteúdo & $\begin{array}{l}\text { É a interação mental entre o aluno e o conteúdo da } \\
\text { disciplina. Alunos que interagem o conteúdo por mais } \\
\text { tempo apresentam melhores notas no final da disciplina e } \\
\text { desenvolve melhor o aprendizado do conteúdo. }\end{array}$ \\
\hline
\end{tabular}

Adaptado de João Mattar (2011)

Chat, fórum, wiks, dão voz aos sujeitos no AVA, dessa forma, professor e aluno não se encontram para transmitir e assimilar conteúdos, mas sim para a construção de conhecimento. A interatividade, dessa forma, pressupõe as possibilidades de interação com os materiais e com o AVA para encontrar informações. Consequentemente, a interação no AVA ocorre entre aluno-professor, aluno-tutor, aluno-aluno, aluno-material educacional didático.

\section{METODOLOGIA}

Este estudo configura-se como uma pesquisa qualitativa (LUDKE e ANDRE, 1986), fundada em cunho exploratório, apoiada pelo estudo de caso segundo Yin (2005). 


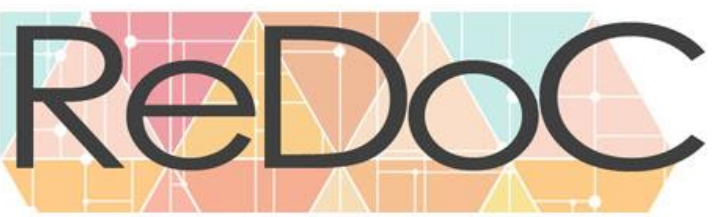

Revista Docência e Cibercultura

Envolvendo estudo bibliográfico e documental. A coleta de dados foi realizada a partir da aplicação de um questionário com 13 perguntas, das quais três com respostas abertas (dissertativas), com o objetivo de analisar as interações e interatividade no ambiente virtual de aprendizagem Moodle de uma disciplina do curso de pedagogia- UAB, na percepção dos alunos. Buscando responder: $\mathrm{O}$ que motiva o aluno a interagir no AVA e quais as ferramentas e elementos disponíveis no AVA que influenciam a interação virtual?

Possibilitando perceber a influência que a interação possui sobre o processo de ensino-aprendizagem. O contato com os alunos ocorreu primeiramente por $e$-mail, com o questionário anexo a mensagem enviada, e-mails obtidos por intermédio do Núcleo de Educação a Distância. O segundo encontro aconteceu para recolher as assinaturas do Termo de Consentimento Livre e Esclarecido, por meio do encontro presencial do Polo Maceió. A amostra desse estudo é composta por 13 alunos que responderam o questionário e assinaram o termo. A turma é composta de 20 alunos. O nome da disciplina e do professor não será divulgado para preservar a identidade dos sujeitos envolvidos. O tratamento das informações foi realizado a partir do software Microsoft Excel e formulário Google.

\section{RESULTADOS E DISCUSSÕES}

Segundo Kenski (2004, p. 104) "Interagir com as informações e com as pessoas para aprender é fundamental" e o AVA é o espaço de interação entre os alunos que estão conectados virtualmente com diversas finalidades que vão desde esclarecer dúvidas ao proposito maior que é aprender. Por conseguinte, os alunos do curso analisado consideram que as interações no ambiente virtual enriquecem a aprendizagem. O que é marcado em suas falas, como mostra a tabela abaixo:

Tabela 1: Interação no AVA como recurso para enriquecer a aprendizagem.

\begin{tabular}{|l|}
\hline $\begin{array}{l}\text { A interação no ambiente virtual com a turma, professor e tutor motivam a sua } \\
\text { aprendizagem? }\end{array}$ \\
\hline "Muito e por esse motivo mesmo que aprendo mais". (A1) \\
\hline "Com certeza e ajuda muito nos trabalhos". (A2) \\
\hline
\end{tabular}




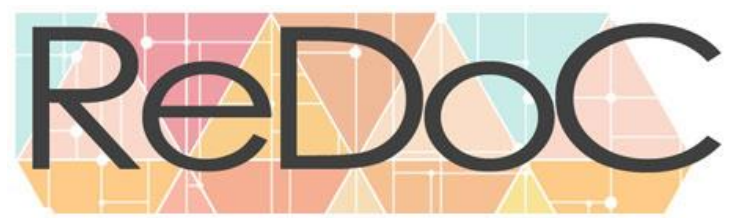

Revista Docência e Cibercultura

\begin{tabular}{|l|}
\hline "Sim, tiro dúvidas e também ajudo a esclarecer questões sobre as quais tenho \\
conhecimento". (A3) \\
\hline "Sim, isso viabiliza a aprendizagem". (A4) \\
\hline "Sim, soma aprendizagem e assim tiro dúvidas". (A5) \\
\hline "Sim, pois tiramos nossas dúvidas". (A6) \\
\hline
\end{tabular}

Fonte: dados da pesquisa.

A troca entre os envolvidos, as diferentes ideias e esclarecimento faz com que ambos acabem se ajudando. Isto é, o compartilhamento de conhecimentos enriquece a interação no AVA e reflete na construção do aprendizado. Essa interação estimula os alunos a participarem mais das discussões levantadas no AVA. Contudo, parte dos alunos relataram precisar de comunicação presencial para uma maior interação com a turma, ou ainda, conforme um dos alunos: "É sempre bom ter o contato pessoal o "olho no olho" (A2). A partir disso, é possível perceber que os encontros presenciais no polo são necessários para esses alunos. Quando questionados sobre os benefícios da interação no AVA, as respostas foram as seguintes:

Tabela 2: Benefícios da interação no AVA.

\begin{tabular}{|l|}
\hline \multicolumn{1}{|c|}{ Benefícios da interação no AVA na percepção dos alunos } \\
\hline "Os fóruns são bem uteis e vêm agregar informações e troca de conhecimento." (A3) \\
\hline "Estimula a estudar". (A7) \\
\hline "A interação é relevante para o processo de aprendizagem, pois expomos nossas \\
opiniões e aprendemos também com nossos colegas". (A8) \\
"Ver e compreender as opiniões dos colegas, pois nos ajuda a tirar dúvidas sobre o \\
conteúdo". (A9) \\
"Contribuir e receber contribuições dos colegas que compartilham das mesmas \\
dificuldades nas disciplinas". (A11) \\
\hline "Troca de conhecimentos, facilidade no estudo, espirito de união entre as turmas." \\
(A12) \\
\hline "Em nós ajudar na compreensão dos estudos". (A13) \\
\hline
\end{tabular}

Fonte: dados da pesquisa.

Como vimos nas respostas, a interação beneficia a aprendizagem aproximando os alunos que estão em espaço e tempo distintos. Promovendo questionamentos e ajudando a compreender melhor o conteúdo estudado, a interação constitui, portanto, um canal de expressão e troca mútua. Na percepção dos alunos, os principais benefícios da interação é a troca de informações, a comunicação com os colegas. 


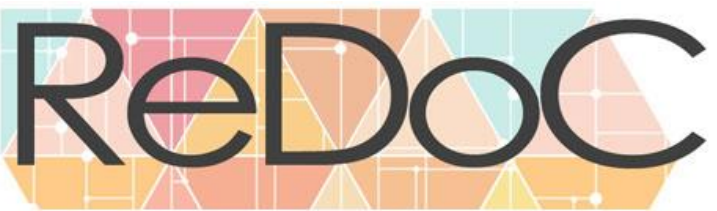

Revista Docência e Cibercultura

Outro ponto importante levantado nas respostas do questionário é a linguagem utilizada no AVA que de acordo com Marquesi e Araújo Jr (2008, p. 363): “É mais informal do que a linguagem utilizada nos textos escritos em geral, na medida em que as pessoas se utilizam dela como estratégia de aproximação, mas possui as peculiaridades do texto escrito, isto é, pode ser planejada, pensada, reformulada. "Ao responderem sobre a linguagem utilizada pelo professor e tutor facilita o entendimento, a maioria acredita que a linguagem é clara e objetiva, (4) apresentam dificuldades em entender o que está sendo solicitado e (2) percebem a linguagem como muito formal, acarretando em um ambiente impessoal.

O estudo analisou também, os elementos que podem vim a bloquear as interações no AVA. Como resposta os alunos elencaram: a falta de conexão com a rede, a manutenção da plataforma ou do provedor local, o sistema fora do ar e a demora no feedback do tutor. Contudo, nota-se que os bloqueios na interação na percepção desses sujeitos estão relacionados ao acesso ao AVA. Na concepção de Xavier e Araújo (2008) as ferramentas disponíveis no AVA, nada farão se não haver interação de qualidade entre os envolvidos no processo. E, apesar do Moodle possui várias ferramentas que promovem a interação, é necessário que haja motivação para que isso aconteça.

Entre os motivos e interesses que levam o grupo a interagir no AVA estão: Dúvidas sobre conteúdo, avaliação e atividades (11), comentar e refletir sobre o conteúdo da disciplina, trazendo questionamentos e refletindo sobre o conteúdo, junto aos demais (7), colaborar com o grupo, comentando as atividades da turma, com o intuito de construir o conhecimento de forma colaborativa (6), colaborar anexando materiais e conteúdo interessantes a disciplina (5).

Além disso diversas ferramentas de comunicação e interação são utilizadas. Predominam, contudo: email (11), mensagem no AVA em atividades (12), fórum (6), chat (5), videoconferência (5) e telefone (4). Todavia, as ferramentas de interação preferidas pela turma são o Whatsapp (13), fórum (4) e o email (3). O grupo no Whatsapp da turma é o principal canal de comunicação, usado apenas pelos alunado. É importante, 


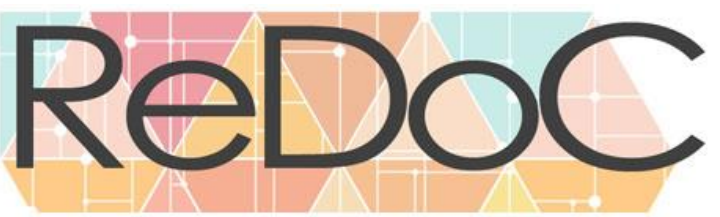

Revista Docência e Cibercultura

esclarecer que apesar do e-mail ser mais utilizado, além do fluxo de mensagens em atividades, o fórum é o recurso mais usado como ferramenta de interação dentro do AVA.

\section{CONSIDERAÇÕES FINAIS}

Ao ponderar sobre o objetivo deste estudo, a analise permitiu perceber que há interação e interatividade no AVA- Moodle da disciplina em questão. Os dados apontaram que as ferramentas utilizadas para a interação online são o e-mail, o fórum, os espaços destinados as atividades e o chat, bem como que o aplicativo Whatsapp é a mídia preferida pelos alunos para comunicação e interação.

Com relação aos elementos que influenciam a interação está o desejo em colaborar com a turma compartilhando materiais e recursos que enriquecem as discussões no AVA e que um dos principais motivos que levam a interação é sanar dúvidas e comentar as atividades e trabalhos do grupo. Tais aspectos configuram a motivação. Bem como, o grupo acredita que a interação influencia positivamente na aprendizagem. O AVA da disciplina oferece ferramentas que propiciam a interação, porém o grupo interage mais por meio do aplicativo Whatsapp, recurso preferido pela turma para comunicação com relação a disciplina, porém essa interação acontece sem mediação do professor.

\section{REFERÊNCIAS}

BEHAR, P. A. Modelos pedagógicos em Educação a Distância: um olhar a partir das competências. 2013.

BELLONI, M.L. Educação a Distância. $2^{a}$ edição. Campinas, SP: Autores associados. 2001.

BENTES, R. F. A Avaliação do Tutor. In: LITTO, F. M.; FORMIGA, M. M. M. (Org.). Educação a Distância: o estado da arte. São Paulo: Pearson Education do Brasil, 2008, v. p. 166-170.

FIGUEIREDO, C. G. Silva; Figueiredo, V. F. Ambiente Virtual de Aprendizagem: comunicação, interação e afetividade na EAD. Aprendizagem em EAD, v. 1, p. 1-16, 2012. 


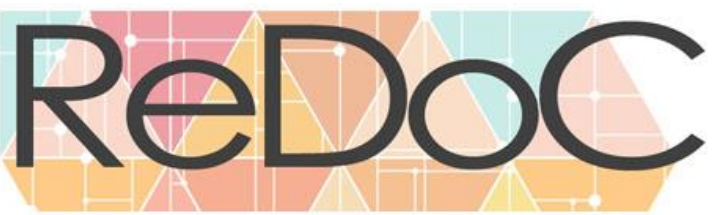

Revista Docência e Cibercultura

ILLERA, J. R. Os Conteúdos em Ambientes Virtuais. Psicologia da Educação Virtual. P.137-152.2010.

KENSKI, V. M. (2004). Tecnologias e ensino presencial e a distância. $4^{\mathrm{a}}$ ed. Campinas: Papirus.

LINS, R; MOITA, M. H; DACOL, S. Interatividade na Educação a Distância. In: III Congresso Nacional de Excelência em Gestão, 2006.

MARQUESI, S. C.; ARAÚJO JÚNIOR, Carlos Fernando de. Atividades em ambientes virtuais de aprendizagem: parâmetros de qualidade. In: LITTO, F. M.; FORMIGA, M. M. M. (Org.). Educação a Distância: o estado da arte. São Paulo: Pearson Education do Brasil, 2008, v. p. 358-368.

MATTAR, João. Interações em Ambientes Virtuais de Aprendizagem histórico e modelos.

Disponível

em: $<$

http://www4.pucsp.br/pos/tidd/teccogs/artigos/2014/edicao_9/4-

interacoes_ambientes_virtuais_aprendizagem-joao_mattar.pdf $>$. Acesso em: 23 de julho de 2017.

PEREIRA, A. T. C.; SCHMITT, V.; DIAS, M. R. A. C. Ambientes Virtuais de Aprendizagem. $2007 . \quad$ Disponível em: https://www.researchgate.net/publication/265141854_Ambientes_Virtuais_de_Aprendi zagem>. Acesso em: Acesso em 20 de julho de 2017.

SAMPIERI, R. H.; COLLADO, C. F.; LUCIO, P. B. Metodologia da pesquisa. 5. ed. São Paulo: McGraw-Hill. 2013.

SANTOS, E. Formação de professores e cibercultura: novas práticas curriculares na educação presencial e a distância. Revista da FAEEBA - Educação e Contemporaneidade, v. 11, n. 17, p. 113-122. 2002.

SILVA, Marco. Que é interatividade. Entrevista concedida em dezembro de 2010. Disponível em: < https://www.youtube.com/watch?v=ShRODbkFIJ0>. Acesso em 23 de julho de 2017.

XAVIER, K; ARAUjO, L. Ambientes Virtuais de Aprendizagem: A Interação do Estudante com as Diversas Ferramentas. In: $14^{\circ}$ Congresso Internacional ABED de Educação a Distância, 2008, Santos - SP. Mapeando o Impacto da EAD na Cultura do Ensino Aprendizagem, 2008.

YIN, R. K. Estudo de caso: planejamento e métodos. 5. Ed. Porto Alegre: Bookman, 2015. 